\title{
Vers de nouveaux outils pour les Digital Studies
}

L'exemple d'une plateforme d'annotation collaborative de vidéo utilisée pour l'analyse de la créativité

\section{Vincent Puig et Yves-Marie L'Hour}

\section{(2) OpenEdition}

\section{Journals}

Édition électronique

URL : http://journals.openedition.org/anthrovision/628

DOI : 10.4000/anthrovision.628

ISSN : 2198-6754

Éditeur

VANEASA - Visual Anthropology Network of European Association of Social Anthropologists

Référence électronique

Vincent Puig et Yves-Marie L'Hour, "Vers de nouveaux outils pour les Digital Studies », Anthrovision [En ligne], 2.1 | 2014, mis en ligne le 15 septembre 2014, consulté le 30 avril 2019. URL : http:// journals.openedition.org/anthrovision/628; DOI : 10.4000/anthrovision.628

Ce document a été généré automatiquement le 30 avril 2019.

(c) Anthrovision 


\title{
Vers de nouveaux outils pour les Digital Studies
}

L'exemple d'une plateforme d'annotation collaborative de vidéo utilisée pour l'analyse de la créativité

\author{
Vincent Puig et Yves-Marie L'Hour
}

\section{L'enjeu des Digital studies}

1 La plateforme d'annotation collaborative développée et utilisée dans le cadre du projet ANR Tic Tac s'inscrit pour l'Iri dans une réflexion théorique plus large sur la refondation épistémologique des disciplines à l'ère du numérique. La thèse soutenue à l'iri et dans les ouvrages de B. Stiegler pose en effet que cette mutation ne pose pas seulement des questions de méthode et d'instruments de travail mais que se joue en fait ce que nous sommes tentés d'appréhender comme une «rupture anthropologique» induite par la numérisation au sens où celle-ci modifie en profondeur ce que Simondon appelait le processus d'individuation psychique et collective ${ }^{1}$ et que Leroi-Gourhan analysait comme un processus d'extériorisation ${ }^{2}$. Cette mutation impacte de manière visible les modes de collaboration et d'éditorialisation et les pratiques sociales. Pour l'Iri, les Digital studies s'inscrivent d'abord dans un nouveau contexte de l'économie de la contribution où la figure de l'amateur devient centrale y compris dans de nombreux projets scientifiques associant les chercheurs et les communautés d'amateurs dans le contexte désigné sous le terme d'Open science. L'analyse de tels projets ${ }^{3}$ montre aussi l'importance de l'organologie numérique au centre de ces processus, sans laquelle aucune grammatisation ${ }^{4} \mathrm{du}$ savoir n'est possible, et par conséquent aucune répartition des tâches n'est envisageable, organologie pensée à l'Iri comme outil de transindividuation.

2 Plus concrètement, cette organologie numérique repose nécessairement selon nous sur un appareil de métadonnées qui doit combiner les approches top-down (taxonomies) et bottom-up (folksonomies), question clé de la convergence actuelle du Web sémantique et $\mathrm{du}$ web social. Ces métadonnées sont à présent l'élément clé d'une nouvelle industrialisation du savoir, non seulement pour Google et les grandes puissances 
industrielles contemporaines mais aussi pour tous les producteurs de savoir dans et hors de l'université. Elles sont aussi à la base des outils collaboratifs tels que ceux que nous présentons dans cet article mais aussi les fondements de nouvelles interfaces sensorimotrices permettant des modes de perception active que le numérique, tel un pharmakon, peut aussi court-circuiter.

Le logiciel "Lignes de temps" à la base de la plateforme que nous présentons ici, a été pensé dans ce cadre conceptuel en s'appuyant par ailleurs sur la théorie des rétentions et des protentions chez Husserl étendue par Stiegler. L'outil est en fait un gestionnaire de métadonnées temporalisées qui a d'abord été utilisé pour la création de discours critiques sur des œuvres cinématographiques. Dans le cadre du projet TicTac, il a été intégré à une plateforme collaborative permettant l'analyse temporellement située des processus de créativité à partir des films réalisés tout au long du projet (séances de brainstorming, entretiens individuels, réunions de travail, visites des ateliers techniques de conception de l'objet...)

\section{Présentation du logiciel Lignes de temps}

4 Le logiciel "Lignes de temps" met à profit les possibilités d'analyse et de synthèse offertes par le support numérique. Inspirées par les «timelines» ordinairement utilisées sur les bancs de montage numérique, Lignes de temps propose une représentation graphique d'un film ou de n'importe quel autre contenu vidéo, révélant d'emblée, et in extenso, son découpage. Lignes de temps offre en cela un accès inédit au film en question, en substituant à la logique du défilement contraint qui constitue l'expérience de tout spectateur de cinéma, et pour les besoins de l'analyse, la «cartographie» d'un objet temporel. Aussi, en sélectionnant un segment d'une ligne de temps, l'utilisateur a-t-il accès directement au plan ou à la séquence correspondante dans le film, séquence qui peut être décrite et analysée par des commentaires textuels, audio, vidéo, ou documentée par des images ou des liens Internet.

5 Il est par exemple possible de parcourir un film plan par plan (séquençage automatisé au moyen d'une autre application, shotdetect, développée à l'IRI), mais également selon d'autres paramètres à partir desquels il est possible de construire d'autres lignes de temps et d'initier d'autres cheminements d'explorations, en fonction des échelles de plan, des mouvements de caméra, des entrées et sorties de champ et potentiellement de toute forme objectivable. Autant de manières de multiplier les approches, mais plus encore de visualiser, par comparaison et combinaison des critères de pertinence, des effets de sens, d'actualiser, par exemple, des récurrences et des symétries.

\section{L'appareillage des pratiques « amateurs »}

D'ores et déjà des historiens, des critiques, des théoriciens, des étudiants d'écoles d'art et de cinéma utilisent cet outil pour vérifier des hypothèses qu'ils avaient formulées, pour en explorer de nouvelles et permettre, à travers ce travail, l'expertise, la critique, l'enrichissement, voire le détournement de ces dispositifs. Bénéficiant de l'expérience d'une communauté experte en matière d'analyse cinématographique, de nouveaux cercles d'amateurs développent progressivement de nouvelles « pratiques » de "Lignes de temps". C'est notamment le cas des professeurs de l'enseignement primaire et secondaire (écoles, collèges, lycées) abordant l'étude du cinéma et plus largement des cinéphiles 
désireux de concrétiser leur appréhension d'un film, leurs souvenirs, leurs impressions, leurs réflexions, sur un support approprié à ces contenus temporels.

\section{Enjeux : la production de regards signés}

7 Concept hérité des «Écoutes signées» développées à l'Ircam à l'initiative de Bernard Stiegler, les Regards signés incarnent le point de vue d'un amateur, qu'il soit critique, réalisateur, enseignant ou élève. S'ils sont construits à l'aide du logiciel Lignes de temps, ils peuvent cependant être publiés sous différentes formes (ou «rendus»). Parmi les formes de rendus possibles, Lignes de temps propose dans un premier temps la visualisation de séquences mises «bout à bout», la comparaison de deux extraits vidéo en parallèle, la lecture d'un hypertexte dont les liens déclenchent les séquences évoquées, la visualisation de séquences avec commentaire audio synchronisé...

8 Les travaux des résidents de l'IRI ont d'ores et déjà révélé de nouvelles possibilités d'exploration de la construction narrative, des parcours thématiques à travers plusieurs films, une mise en lumière des relations entre cinéma, photographie et peinture, voire une analyse génétique de l'œuvre ou la mise en évidence du changement de statut des images selon le contexte historique, culturel, social ou politique de leur production et/ou de leur réception.

9 La production de discours critiques sur les processus de création mis en œuvre dans le contexte du projet TicTac s'inscrit dans la droite ligne de cet axe de recherche.

Figure 1 Regard signé sur double-écran annoté pour le film Alumbramiento de Victor Erice

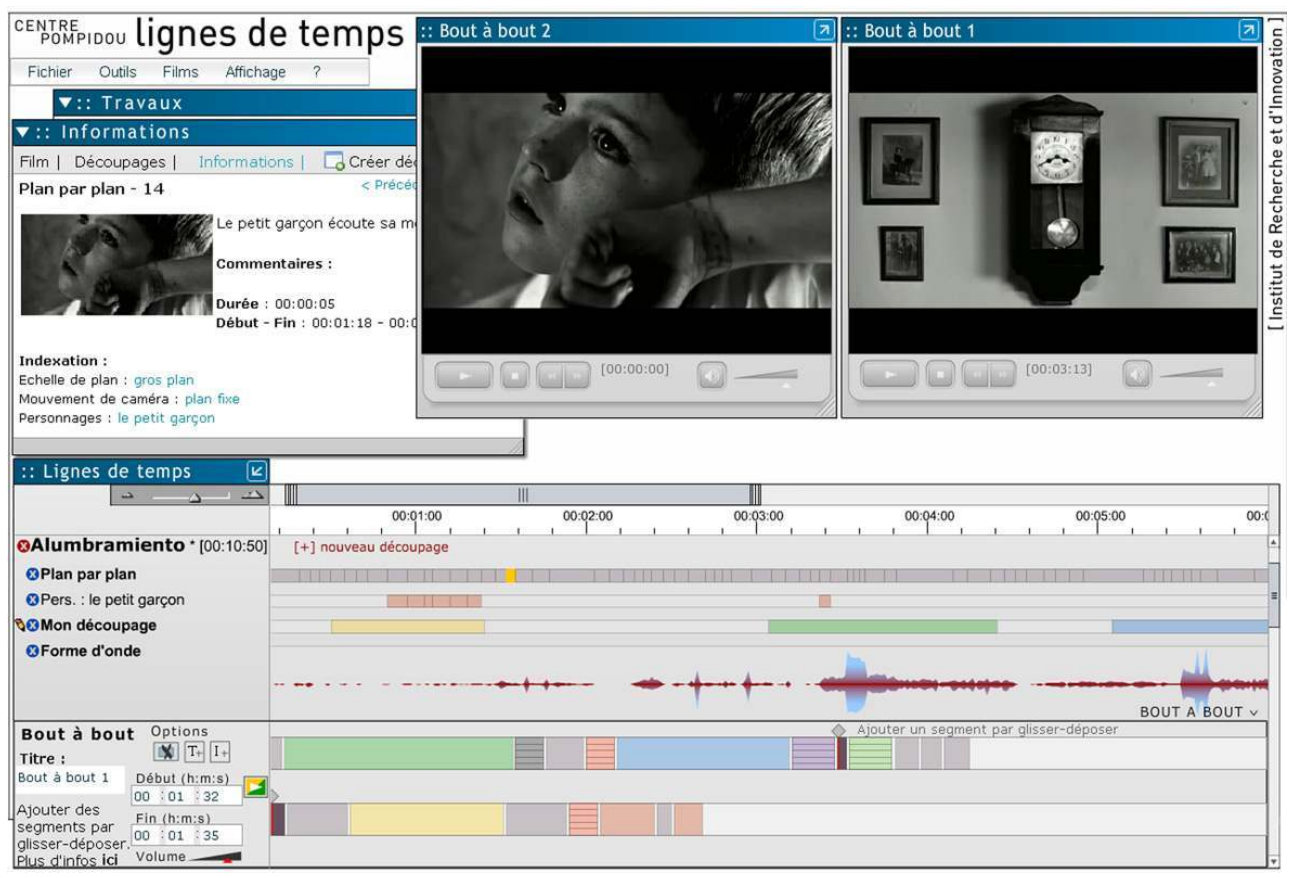




\section{Contexte et enjeux du projet Tic Tac sur l'analyse de la créativité}

\section{Objectifs}

10 L'objectif du projet TICTAC est d'analyser les éléments objectifs et subjectifs qui déterminent la dynamique du processus de création depuis sa naissance jusqu'à sa mise en public. Pour cela, nous nous proposions d'étudier scientifiquement à la fois l'évolution des idées, des artefacts et la coopération des différents acteurs au cours de l'élaboration d'une ou plusieurs des créations qui seront développées dans le contexte de l'initiative Garage. C'est donc une histoire microscopique des idées tout au long d'un projet de création que nous voulions dresser, en considérant ces idées comme la médiation effective d'une interaction permanente entre les acteurs et les différentes formes matérielles de cette élaboration.

\section{Originalité}

D'ores et déjà, il faut comprendre que l'objectif du projet TICTAC (projet ANR réunissant le laboratoire CEA LID, le LISTT-CERS et l'IRI) est indissociablement lié à l'initiative Garage, qui lui confère une grande part de son originalité, pour deux raisons. D'une part cette initiative nous permet de mener une recherche sur la problématique de la création sans chercher:

- ni à l'isoler des autres formes qui lui sont apparentées (innovation, découverte, invention, créativité...),

- ni à lui conférer à l'avance un statut (artistique, technologique, scientifique...),

- ni à enfermer préalablement les acteurs de cette création dans une posture ou un rôle (artiste, chercheur, inventeur, ingénieur, technicien...).

D'autre part, cette initiative nous permet de considérer une forme de création qui demande la coopération d'un ensemble d'acteurs pour se réaliser. En effet, les projets technologiques, comme ceux qui sont envisagés dans Garage, demandent la contribution de plusieurs compétences qui sont autant de métiers différents (micro-électronique, transmission, énergie, design...). Si l'on transpose dans le champ de la création artistique cette exigence, nous nous rapprochons de la situation qui prévaut dans le champ du cinéma, par exemple, et nous pourrons nous demander dans quelle mesure le type de création que nous analyserons peut se comparer à ce qui a été compris dans ce domaine particulier.

\section{Intérêt du sujet, enjeux et perspectives}

13 La créativité dans le domaine de l'innovation techno-scientifique s'est vue dotée de quelques-uns des traits traditionnellement attribués à la création artistique, notamment sa singularité individuelle. Assimilée le plus souvent, par les ingénieurs eux-mêmes, au surgissement spontané de l'idée originale, la création a longtemps été restreinte au processus mental d'un sujet, processus que la psychologie cognitive a elle aussi eu tendance à cantonner dans la sphère infra-cerveau. 

l'action, permet désormais d'envisager l'acte créatif comme un acte non pas isolé mais au contraire immergé dans un environnement physique et mental collectivement partagé. L'acte créatif s'inscrit dans la multiplicité des échanges et des coopérations au sein d'un système cognitif que partagent des individus (chercheurs, ingénieurs, artistes, techniciens...) par l'intermédiaire d'artefacts techniques (images, langages, instruments... ).

15 La création elle-même peut ainsi être abordée comme une activité distribuée, celle que partagent les individus entre eux, comme celle qu'ils partagent avec les objets qui génèrent et médiatisent leurs échanges, comme celle enfin que peuvent aussi partager entre eux ces mêmes objets (artefacts cognitifs). Une telle approche d'une «créativité distribuée » nécessite dès lors que soient révisées, d'une part la conception d'une idée créatrice soustraite d'un substrat matériel, d'autre part la conception d'une création qui serait l'unique attribut d'un génie singulier.

\section{Fonctions techniques questionnant la méthodologie d'analyse globalement}

16 La technique est au cœur des enjeux de créativité et de développement de l'imaginaire que nous cherchons à mettre en évidence dans ce projet. Tout d'abord dans la mesure ou toute la pensée créatrice des acteurs est plus ou moins conditionnée ici par l'objectif de production d'un objet technique innovant. Les participants du monde technologique ayant une conscience accrue de ces contraintes, les participants observateurs (sociologues) à un degré moindre mais étant également impliqués dans le processus de création, ce qui n'a pas induit des biais problématiques fondamentaux. En revanche, l'appareil technologique de second niveau à savoir le dispositif d'enregistrement des séances et surtout d'analyse de ces séances a posé selon nous des questions méthodologiques nouvelles et problématiques.

17 En effet, sur la plateforme permettant de revenir sur les enregistrements, chaque utilisateur est identifié. Il a la possibilité de créer plusieurs projets d'annotations de films, de les laisser privé, par exemple un projet avec seulement des notes et impressions personnelles, ou de le rendre public, par exemple un travail plus abouti et présentant un point de vue argumenté. La version de Lignes de temps en ligne possède des fonctionnalités développées à partir de la version hors ligne : création de nouvelles lignes d'annotations, duplication de lignes et d'annotations, travail sur les nuages de tags, prise de notes, bout à bout, etc. Sur chaque projet, un utilisateur peut travailler sur un ou plusieurs contenus vidéo.

18 L'aspect collaboratif se situe en aval d'un projet publié. Une fois public, un projet peut être copié et approprié par un autre utilisateur. Celui-ci peut alors modifier, contredire, compléter les annotations précédemment écrites, et publier à son tour sa participation. Ainsi de suite, un dialogue autour du ou des films se met en place. Tic Tac. L'utilisation de ces comptes permettait de tracer les annotations et les contributions apportées par chaque utilisateur de la plateforme.

D'emblée le support épistémologique utilisé, à savoir l'enregistrement vidéo, pose problème. Beaucoup de sociologues et d'anthropologues utilisent à présent la vidéo, mais 
dans la plupart des cas, ils en font une transcription textuelle qui sera la base de référence de l'analyse. En proposant un autre support pour l'analyse, à savoir la vidéo annotée (grammatisée), la plateforme impose de nombreux changements méthodologiques pour ne citer que les plus évidents : la pré-éminence nouvelle du nonverbal, l'apprentissage de nouvelles formes de lecture (navigation vidéo) et surtout d'écriture (annotation) qui sont impossibles à pratiquer sans un travail d'indexation préalable. Ceux des participants qui sont allé jusqu'à une indexation fine de la vidéo ont pu en saisir l'intérêt ne serait ce que par l'utilisation du moteur de recherche intra-vidéo (sorte d'index fabriqué automatiquement au fur et à mesure de l'indexation. Mais la fonction technique qui n'a pas été suffisamment développée est celle de l'annotation collaborative. Ici, comme nous l'exposions au chapitre 1, le savoir individuel s'appuie nécessairement sur l'indexation produite par les autres participants. S'ils n'ont pas procédé à l'annotation d'un segment vidéo donné, le segment en question pourra passer inaperçu ou n'apparaitra que pour certaines requêtes, certains mot-clés.

\section{Mise en œuvre : objectivation, subjectivation, communautisation}

21 L'outil "Lignes de temps" a été utilisé dans le cadre du projet Tic Tac à trois niveaux spécifiques :

- Analyse objective

- Analyse subjective

- Analyse contributive (ou communautaire)

Dans le cadre par exemple de l'analyse de la séance créativité du 4 novembre 2009, le niveau objectif est matérialisé par les deux premières Lignes de temps respectivement intitulées « Intervenants » et "Séquences ». Le premier découpage permet d'identifier les prises de paroles des participants, le second de visualiser immédiatement les différents temps (exercices, échanges collectif ou en binômes, etc.) de la séance de brainstorming.

Aux critères de segmentation précédemment décrits et qualifiés «d'objectifs», les praticiens du logiciel peuvent ainsi ajouter leurs propres lignes subjectives en s'appuyant ou non sur les découpages disponibles ou en constituant des groupes de lignes en fonction de critères et de finalités qui leurs sont propres. L'annotation subjective peut dépasser la dimension du visible, porter sur l'analyse du hors champ ou souligner l'émergence technique, narrative ou émotionnelle d'une idée - elle est subjective en cela qu'elle se fonde, non plus sur le déroulé de la séance filmée elle-même, mais sur la rencontre entre le film de la séance et le regard de celui qui l'analyse.

L'outil propose pour ce faire, de poser des marqueurs ou de définir des segments le long des «lignes de temps», puis de leur attacher une annotation en texte libre, des mots-clés gérés par une base de données partageable entre une multitude d'utilisateurs, ou, dans des développements futurs, en faisant appel à des thésaurus (vocabulaires contrôlés) ou des arborescences thématiques.

L'annotation peut aussi prendre la forme d'un commentaire audio enregistré directement via le logiciel ou par synchronisation de documents (fichiers audio, textes, photos, vidéos, URLs).

Troisième enjeu du développement de l'outil Tic Tac, la «communautisation » permet aux participants du projet de partager leurs lignes de temps avec le groupe. La ligne de temps constitue dans ce cas un instrument organisé de dialogue synchronisé aux séances 
filmées. Les lignes de temps des différents contributeurs peuvent en effet être échangées, superposées ou modifiées par les uns ou les autres indépendamment du site collaboratif lui-même.

\section{Médiation technique du processus de création dans un contexte d'innovation technologique}

Le débat classique art et science trouve son renouveau actuel sous la forme de la problématique création artistique et innovation technologique. La médiation technique numérique refonde là aussi ce rapport sous la double contrainte:

D'une part, du progrès quantitatif des technologies qui a produit une rupture qualitative dans l'accès aux moyens de production et de diffusion de la création. Cette rupture a enrichi considérablement les formes reconnues de l'art, mais elle a aussi permis l'apparition de modalités entièrement nouvelles, dont la plus connue est certainement l'art numérique.

D'autre part, le monde industriel lui-même s'est retrouvé confronté à une injonction d'innovation sans précédent, face à laquelle il a cherché des ressources en dehors de son cadre de pensée traditionnel. C'est ainsi que tous les modes de création en général et les méthodes de créativité en particulier ont trouvé très récemment un espace de bienveillance sinon de développement dans le monde naguère fermé de l'industrie.

Dans le projet Tic tac nous nous sommes interrogés sur ces rapports entre création et innovation technologique qui pouvaient encore sembler évidents à l'époque de Léonard de Vinci ou l'organologie utilisée pour l'art et pour la science se confond. C'est aussi le cas de manière plus contemporaine dans le développement des fablabs et des nouvelles pratiques amateurs ou encore avec l'exemple de Gérard Hass, un ingénieur de la société Philips, qui fabriquait dans les années 70 des statuettes de métal poli dont les yeux, constitués des tout premiers afficheurs électroniques, s'illuminent, par exemple suivant l'intensité des bruits ambiants.

31 Dans tous ces exemples comme dans le projet Tic Tac et son organologie numérique spécifique, on observe qu'une fois passée l'étape d'appropriation du système technique proposé et partagé, les acteurs développent des processus créatifs, qui sont proches des processus de création « amateur » au sens où pour créer, il faut aimer, désirer l'objet de sa création et aussi son outil de production.

Ces processus créatifs ont été analysés durant le projet par l'équipe du LISST d'Anne Sauvageot comme relevant selon les participants :

- d'une créativité topodynamique (images d'action, de mouvement)

- d'une créativité sensitivo-perceptive (surfaces, textures)

- d'une créativité narrative (histoires)

- d'une créativité technico-fonctionnelle (couple dispositif/fonction)

- ou d'une créativité syncrétique (combinaison des 4 types précédents)

33 Ces types de créativité sont en fait à la fois des types d'imaginaire et se référent à des outils conceptuels et techniques (les catégories du langage étant à prendre ici comme des catégories techniques). 


\section{Prolongement de la créativité dans l'outil numérique}

$\mathrm{Au}$ delà des types de créativité identifiées, le projet visait également à favoriser leur prolongement sur la plateforme d'annotation voir l'apparition de nouvelles formes de créativité dans cet après coup de l'événement.

Production d'une annotation de référence sur la séance de créativité du 4-11-2009

Une annotation de référence menée selon les principes définis ci-dessus a été menée sur les deux demi-journées de la séance de brainstorming du 4 novembre 2009.

Figure 2 Espace d'accès aux contenus et aux projets publiés

TIC-TAC

ymlh Mon profil Esp. perso Déconnexion

\section{Liste des contenus}

\begin{tabular}{|c|c|c|}
\hline TicTac 2009-11-04 Après-Midal & Ouvrir sous Lignes de Temps & Créer un projet \\
\hline TicTac 2009-11-04 Matin & Ourir sous Lignes de Temps & Créer un projet \\
\hline
\end{tabular}

\section{Liste des projets}

\begin{tabular}{|lll|}
\hline jd : creation matin2 & Ouvrir sous Lignes de Temps & Copier projet \\
TicTac Après Midi & Ouvrir sous Lignes de Temps & Copier projet \\
annotations_Léna & Ouvrir sous Lignes de Temps & Copler projet \\
Séance de créativité: Annotation de référence & Ouvrir sous Lignes de Temps & Copier projet \\
\hline
\end{tabular}

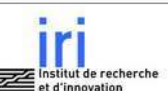

C2010 1R/ / Centre Ponpicou 


\section{TIC-TAC \\ Liste des contenus}

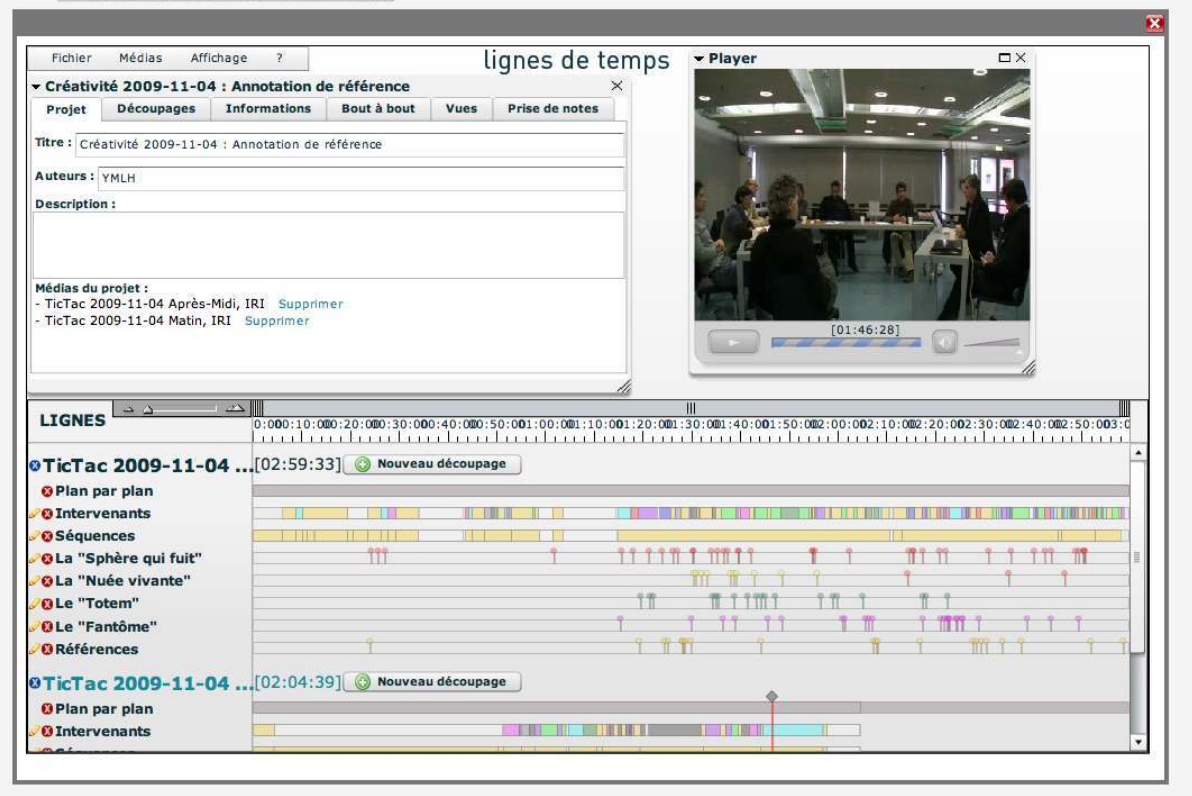

\section{Modes opératoires d'annotation}

\section{Mode opératoire d'annotation individuelle sous Lignes de temps}

Cette méthodologie pouvait être utilisé soit en mode local avec une application Lignes de temps installée sur PC ou Mac, soit sur le site de la plateforme collaborative en mode connecté, dans l'espace personnel de chaque annotateur.

\section{Prise de note}

La fonction «Prise de notes " permet de prendre des notes en temps réel pendant le visionnage de la vidéo. Il est ainsi possible d'annoter immédiatement le média sans avoir à créer de lignes de temps, poser des marqueurs ou des segments puis les décrire, et ainsi faire l'économie d'une étape de retranscription de notes papier sur Lignes de temps : les notes sont directement contextualisées dans le temps même de la vidéo. Il est également possible de développer des commentaires pendant les pauses ou au fil du média, puis de générer une ligne de temps classique à partir de laquelle une annotation détaillée pourra ensuite être générée.

\section{Canevas d'annotation des séances}

Adopter un canevas ou template d'annotation permet aux utilisateurs d'adopter un certain nombre de conventions communes et donc d'être en mesure de dialoguer les uns avec les autres. 

dessous:

- La sphère qui fuit

- La nuée vivante

- Le totem

- Le fantôme etc.

Il était recommandé de procéder à une annotation des séances selon le canevas ci-

Une ligne Intervenants qui permet de distinguer à partir d'un code couleur simple les principaux intervenants de la vidéo;

Une ligne Séquences qui permet de segmenter de façon thématique les différentes phases de la séance (introduction, exercice de créativité, discussion libre, pause...)

Une ligne Références permettant de faire figurer des compléments d'informations, liens hypertextes, références, etc. à l'image des notes de bas de page d'un livre, liés aux citations des participants (par exemple lors de la séance de créativité, des références récurrentes à des œuvres cinématographiques, ou à des cadres culturels) ;

43 Une ligne dite Thèmes ou Effets de contamination permettant de tracer des idées susceptibles de faire l'objet de débat et / ou d'être mis en contraste avec des segments du même enregistrement ou de contenus externes;

La ligne Prise de notes, correspondant aux annotations éventuellement effectuées en temps réel durant la séance de travail peut éventuellement être effacée ou masquée après que son contenu ait été mis en forme dans les autres lignes de temps.

En complément de ces lignes, quatre autres découpages ont été proposés pour l'annotation de référence de la séance de créativité du 4 novembre 2009, conformément aux lignes directrices adoptées dans le livrable 2.1, et en relation avec les quatre propositions ayant émergées à l'occasion de ce brainstorming:

Ces lignes visent à identifier les différentes occurrences de l'idée d'objet à fabriquer en phase d'émergence chez les participants à chacune des phases du brainstorming.

Il est bien entendu possible d'adapter ce canevas en fonction du discours propre à chaque critique ou intervenant, par exemple en créant des lignes supplémentaires dédiées à une perspective particulière d'analyse ou d'usage, ainsi les segments qui pourraient être cités dans un article électronique, et sur lesquels pourra pointer un lien hypertexte en utilisant le permalien identifié au niveau de chaque segment.

B Il est conseillé d'utiliser les segments pour marquer des développements dans la durée et de privilégier l'usage de marqueurs lorsqu'il s'agit de repérer un élément quasi ponctuel dans le discours ou le film (comme cela a été fait pour les quatre lignes thématiques correspondants aux quatre objets pressentis).

49 Une possibilité complémentaire d'annotation offerte par l'outil Lignes de temps est de créer des lignes a priori et de procéder à leur renseignement au fur et çà mesure du déroulé de la séance. Il est ainsi possible de créer une ligne par thématique ou de repérer par un simple marqueur les différentes occurrences d'un concept, d'un mot, d'une thèse, 


\section{Organisation et mise en œuvre pratiques des ateliers d'annotation}

50 Cette méthodologie n'a pas été parfaitement mise en œuvre lors de la première phase du projet Tic Tac, car il était préalablement nécessaire de définir collectivement les axes d'analyse à privilégier à partir des travaux menés par le groupe des sociologues.

51 Le déroulé des ateliers d'annotation était le suivant :

\section{Annotation de la vidéo en mode individuel}

Chaque participant produit cinq à dix annotations sur des séquences de chacun des ateliers ou entretiens, en étant libre du choix des séquences et du point de vue de ses commentaires qui peuvent être liés à sa discipline ou pas (analyse technique analyse sociologique). Une analyse des retours d'expériences pourra se faire fait sous la forme d'entretiens exploratoires, menés (comme ceux déjà effectués lors de cette première phase du projet par Julien Delas et Aurore Bonnet) auprès de certains des participants.

L'objectif était double :

- Tester la facilité d'usage et d'appropriation du dispositif; saisir ce que les utilisateurs auraient aimé faire et n'ont pas pu faire avec Lignes de Temps.

On cherchait également à répondre aux questions : quelle est l'unité significative à partir de laquelle les utilisateurs ont travaillé? Quels sont les détournements constatés du logiciel?

- Comprendre le sens que peut avoir pour son utilisateur le développement, au moyen d'un tel dispositif, d'une approche réflexive sur un contenu.

En particulier, dans le cadre d'un usage "savant », que cela apporte-t-il /change-t-il par rapport au travail de prise de notes sur les retranscriptions des séances? Dans le cadre d'un usage "amateur", quelle utilité spécifique est prêtée par l'utilisateur à l'annotation?

\section{Les commentaires de commentaires en ligne}

Il était prévu une journée de confrontation et une phase de commentaire des commentaires ainsi que la production de « regards critiques » sur les séances. Ces phases n'ont pas pu être menées à bien dans le contexte du projet Tic Tac mais nous présentons ici en dernière partie des exemples produits dans le champs de l'analyse filmique. On pourra aussi se référer au travail d'analyse des usages de cette plateforme mené par l'Institut Télécom'.

\section{Quelques enseignements tirés de l'annotation sur la plateforme}

La plateforme d'annotation collaborative a permis de "visualiser " des hypothèses et ainsi de les confirmer mais aussi parfois de suggérer de nouvelles hypothèses d'analyse. Le traçage des idées dans l'outil montre que sur les 4 thèmes proposés pour la fabrication de l'objet technique, le thème de la sphère s'est imposé au groupe sous l'influence du leader du groupe par des indices précis :

- le rappel des mots d'ordre et de la règle du jeu

- les mots qui font « tilt" 
- les relances

- les figures rhétoriques (changements d'échelle, inversions, glissements, dérapages

- les traits d'humour.

Dans l'annotation réalisée par Julien Delas, il est particulièrement intéressant de naviguer à l'aide du mot clé « censure » ou « autorité ». On tombe effectivement sur une séquence ou le leader du groupe infléchit clairement la dynamique du groupe et on peut tracer avec intérêts les séquences où il impulse sa propre idée d'objet à fabriquer tout en maintenant le discours selon lequel cette idée émane du groupe.

\section{Outils d'expression collaborative et d'éditorialisation}

En guise de conclusion, nous présentons ici quelques exemples de formes d'expression collaborative et d'éditorialisation produites à partir de la plateforme d'annotation Lignes de temps et qui sont le fruit des réflexions méthodologiques menées dans le projet Tic Tac et plus largement des recherches de l'Iri dans le champ des Digital Studies.

Figure 4 Annotation de films en visioconférence (Iri-Institut Télécom)

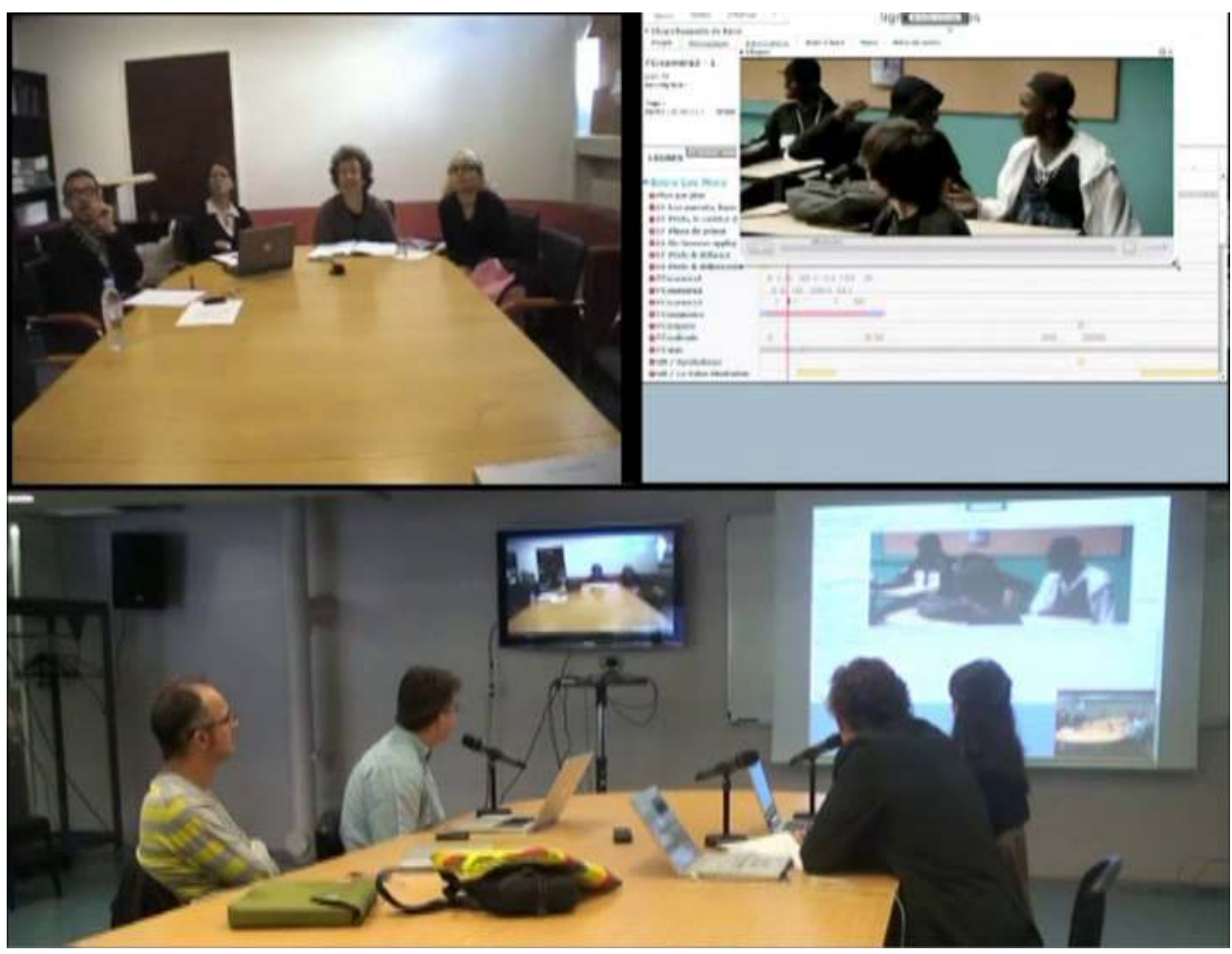

Dans cette illustration on présente un dispositif de visioconférence articulé sur la plateforme Lignes de temps et qui a permis de procéder à une analyse critique collective à distance sur le film «Entre les murs ». L'interface du logiciel présente l'ensemble des lignes produites par les participants et qui sont ensuite discutées, reprises, amendées ${ }^{6}$.

Dans les figures suivantes, on présente différentes modalités éditoriales : player enrichi et moteur de recherche contextuel (fig. 5 et 6), navigation de point de vue en point de vue opposé (fig. 7), cartographie heuristique liée à l'enregistrement d'une conférence (fig. 8), fabrication de mashups (fig 9), articulation d'un texte critique avec des vues sur le logiciel (Fig. 10). 
Figure 5 : Segments vidéo indexés selon la norme media fragment pour France Culture : le moteur de recherche permet d'éclairer les segments identifiés et de les partager sur les réseaux sociaux

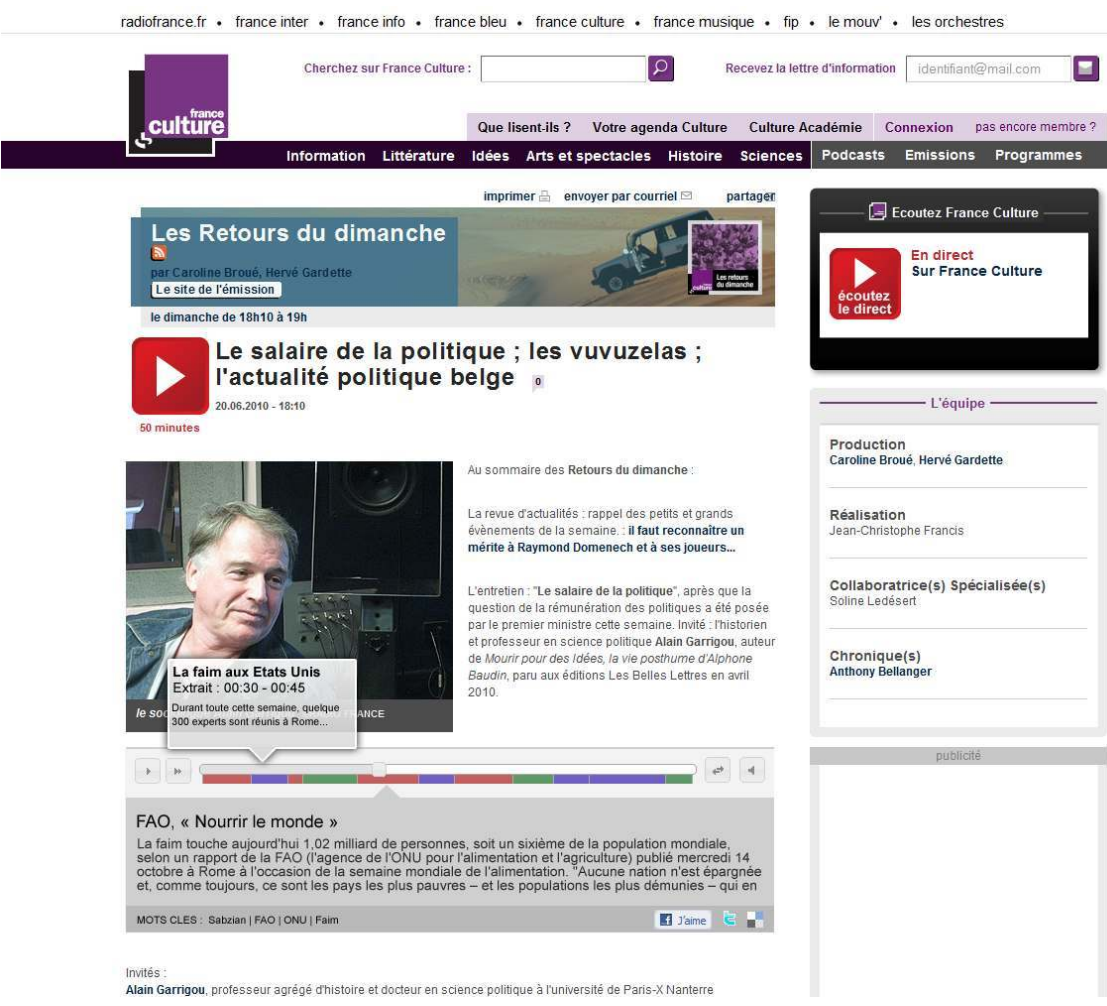

Figure 6 Moteur de recherche dans les segments vidéo et présentation contextualisée des résultats (Ars Industrialis)

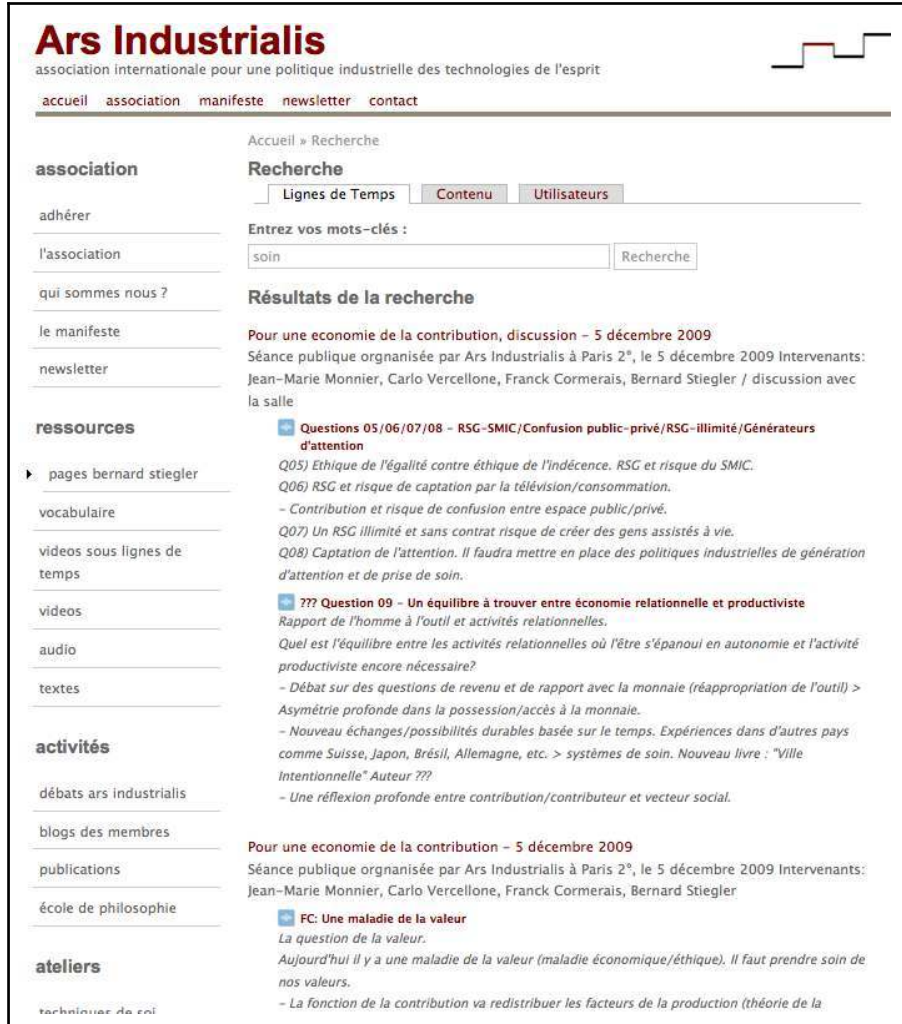


Figure 7 Boussole sémantique : Navigation sémantique (tags) et polémique (couleurs) au fil de l'écoute

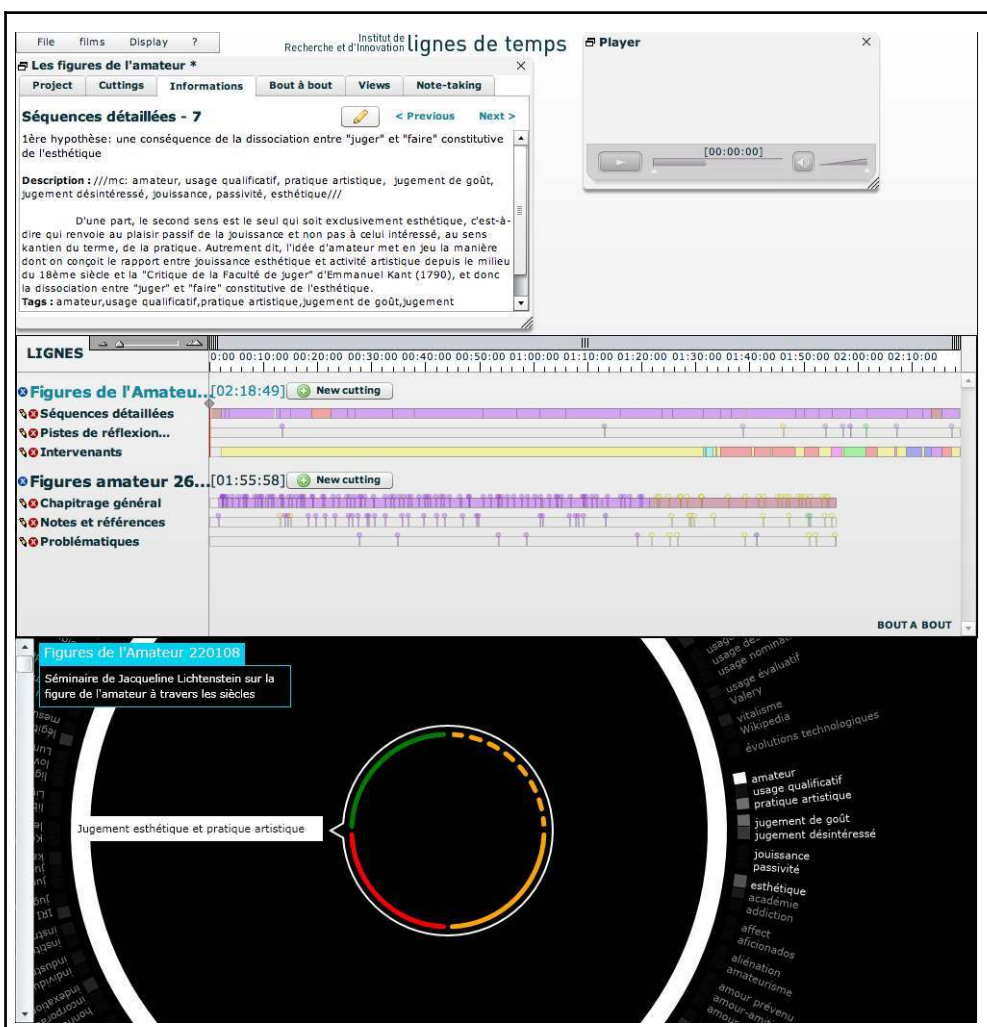


Figure 8 Cartes de navigation heuristique liant les problématiques de la conférence avec l'enregistrement indexé par les tweets des participants

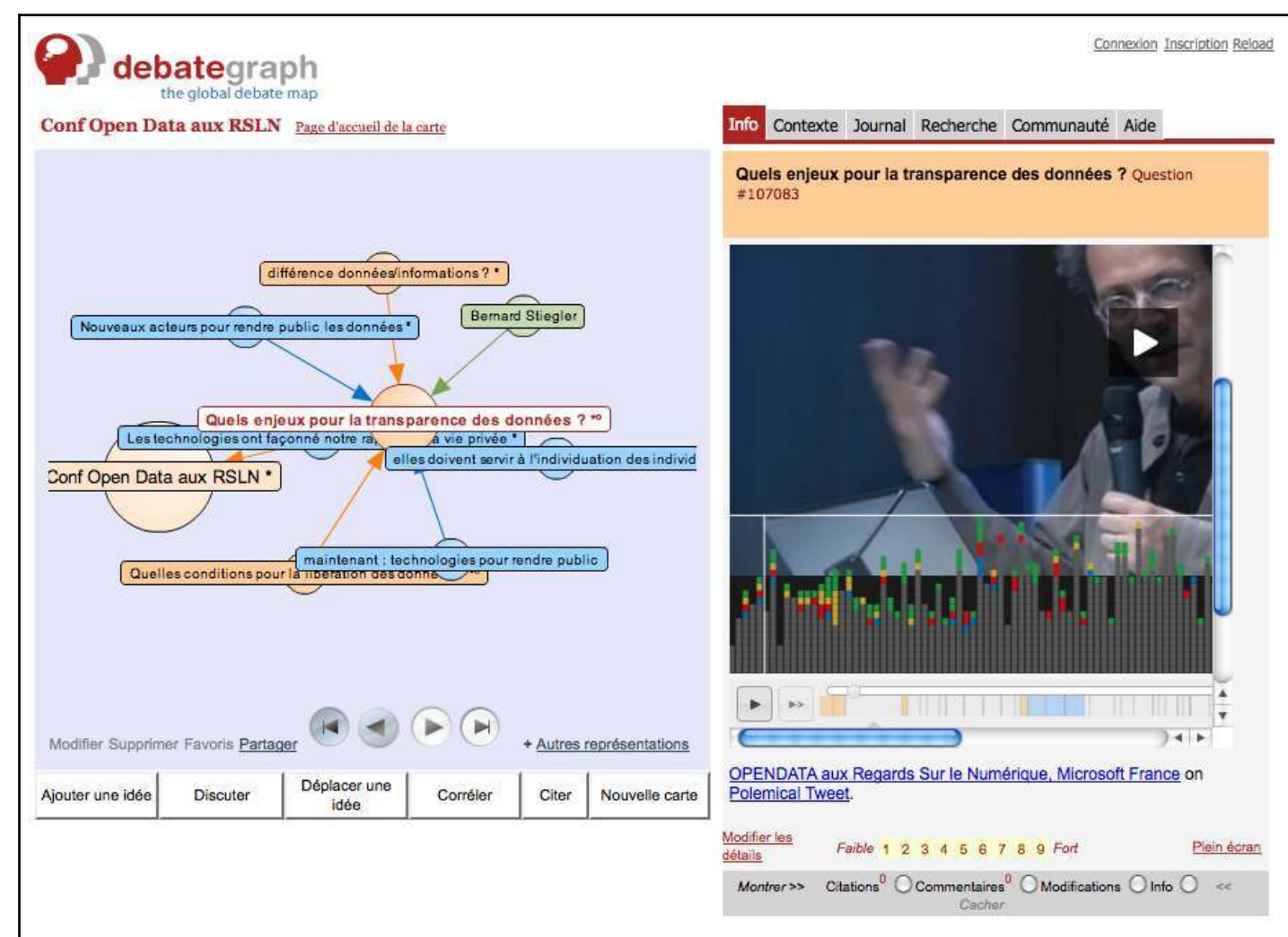

Figure 9 Navigation dans le fonds d'archives de la BPI à partir de mashups créés par les visiteurs

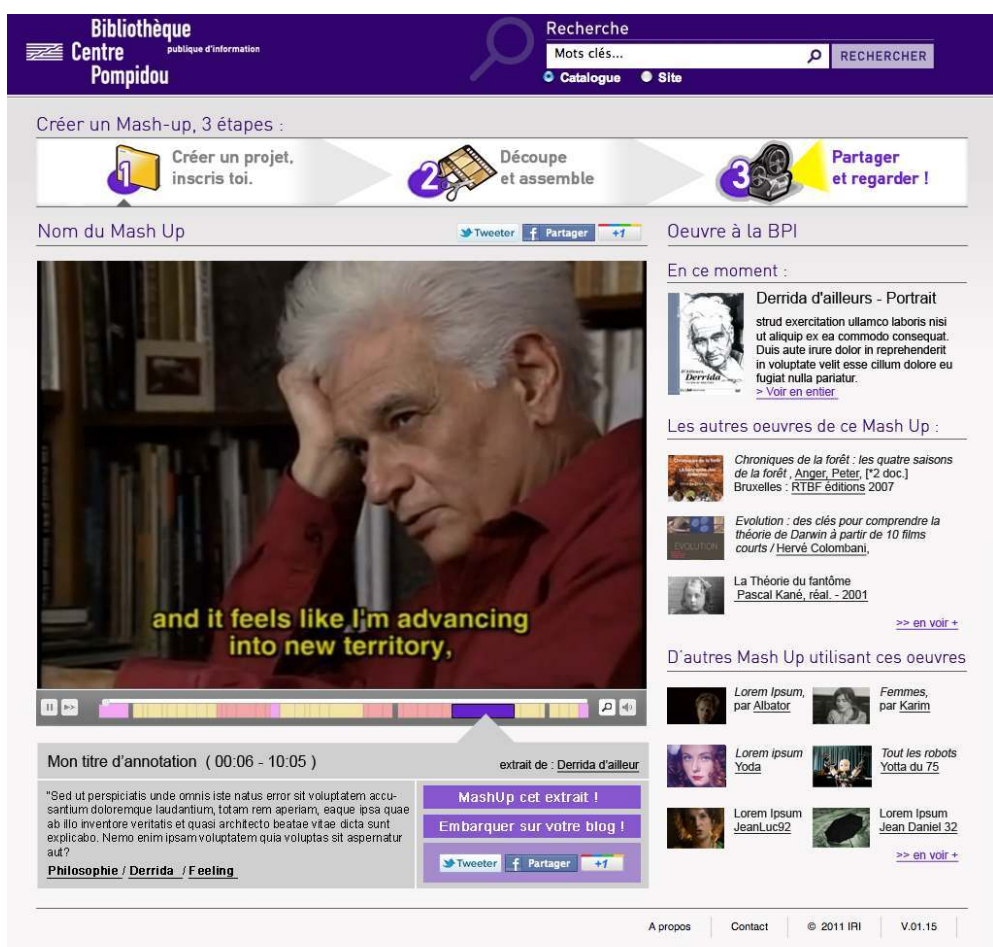


Figure 10 Analyse de Yeelen de Souleyman Cissé par Caroline Archat, Cahier des Ailes du désir

Observer comment les rapports entre la mère et le fils sont filmés : miroirs et jeux de regards

Pour réaliser la mise en lien d'une idée avec les éléments de la mise en scène, la création de découpages, de descriptions et de bouts à bouts s'avère utile.

Dans la perspective d'une démarche qui consiste à se laisser surprendre par les images et le son, on étudie trois plans (segments verts).
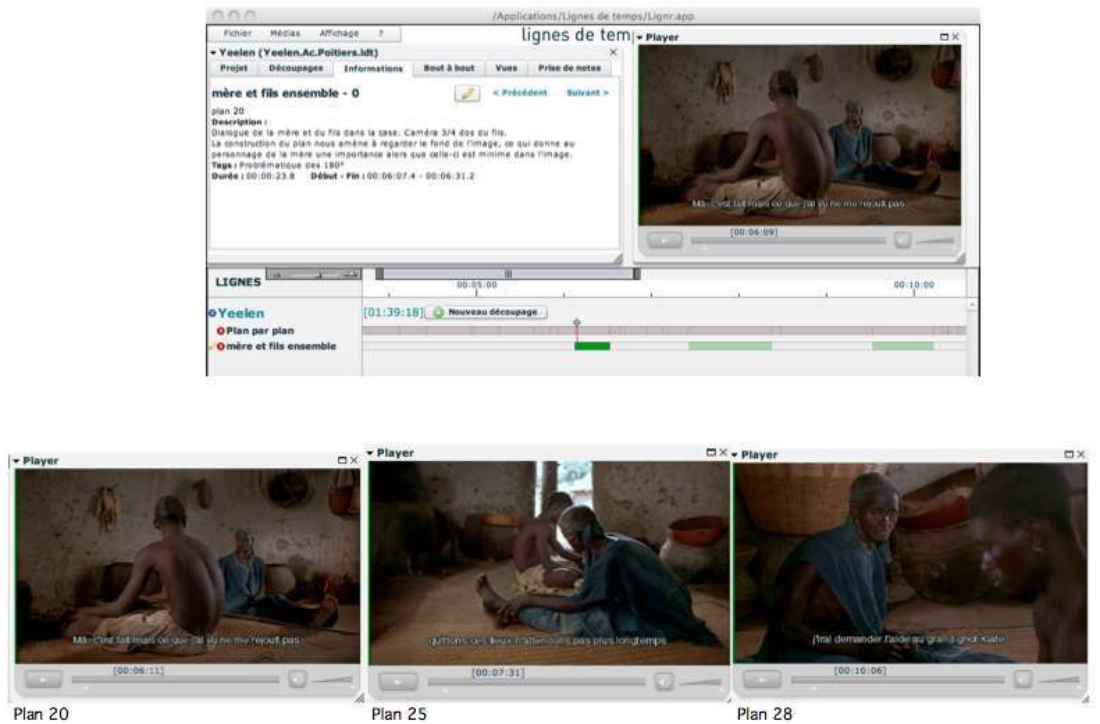

Figure 11 Tagging gestuel de films de danse sur une surface tactile

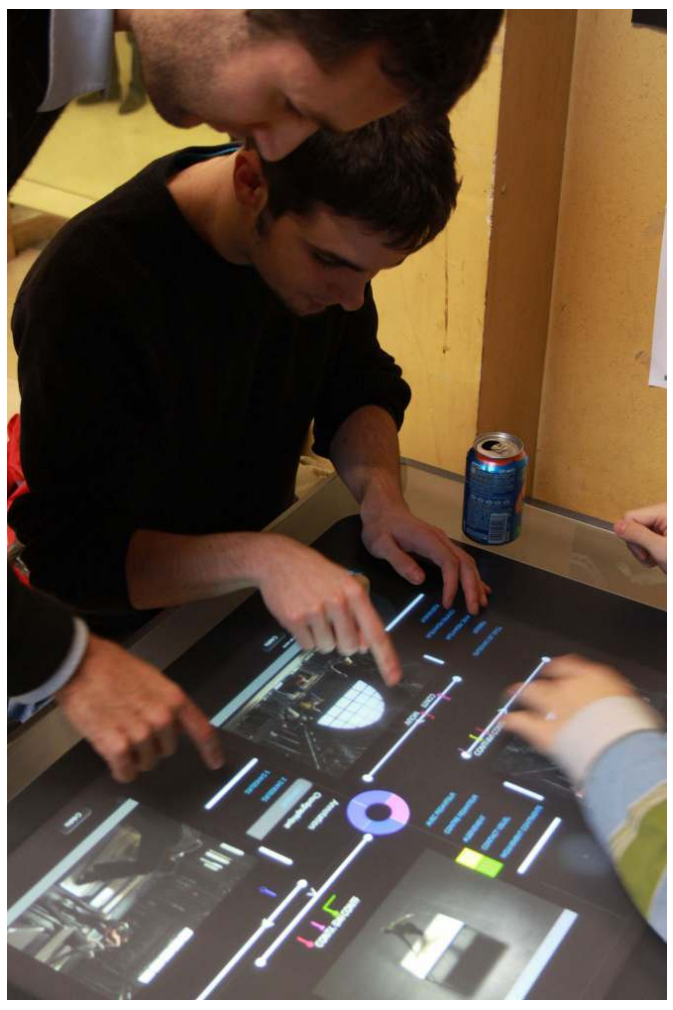

63 Avec le dernier exemple présenté ici (Fig. 11) nous voulons illustrer l'enjeu de la sensorimotricité dans le champ des Digital Studies. En effet, des plateformes telle que celle que 
nous avons présentée dans cet article doivent in fine permettre aux producteurs de savoirs à tous les niveaux de concevoir non seulement des contenus indexés mais dans le même temps, et grâce à l'indexation sous-jacente, des interfaces ouvrant à des formes de perception active indispensables à une bonne appropriation (des instruments de musique pour entendre, des pinceaux pour voir, des outils d'écriture pour bien lire, etc...). La sensori-motricité serait ultimement à considérer comme un parallèle dans le domaine de l'énaction (articulation de la pensée et du corps) à un modèle énactif global articulant individuation psychique et collective, savoir individuels et « intelligences collectives ».

\section{NOTES}

1. Gilbert Simondon, L'individuation psychique et collective, Aubier, 2007

2. André Leroi-Gourhan, Milieu et techniques, Paris, Albin Michel, 1945

3. http://fr.wikipedia.org/wiki/Foldit

4. Sylvain Auroux, La révolution technologique de la grammatisation, Ed. Mardaga 1994

5. Beaudouin V., Fournout O., Ferrarese E. La nouvelle Utopia des médias électroniques : le cas de l'annotation collaborative de films, à paraître.

6. Collaboration Iri-Institut Télécom, projet FUI THD, 2010

\section{RÉSUMÉS}

L'anthropologie, la sociologie et toutes les sciences humaines et sociales se trouvent aujourd'hui confrontées au défi du numérique. Une approche très répandue aux Etats-Unis et dénommée sous le terme générique des Digital Humanities, se réduit souvent à étudier comment les SHS font usage du numérique. Notre approche dans cet article propose dans le cadre général posé par B. Stiegler, non pas seulement d'observer mais aussi de concevoir des outils qui peuvent ultimement permettre de reconsidérer les fondements épistémologiques des différentes disciplines académiques (et pas seulement SHS). Nous prendrons appui dans cet article sur une plateforme d'annotation collaborative de vidéo utilisée dans le cadre du projet ANR Tic Tac (2009-2011) sur l'analyse de la créativité en tentant d'isoler les fonctions techniques de cet outil qui questionnent précisément: la méthodologie d'analyse sociologique ou anthropologique globalement, le processus de création dans un contexte d'innovation technologique (projet Garage du CEA), le prolongement de la créativité dans l'outil d'annotation voir dans des outils d'éditorialisation collaborative expérimentés par l'Iri dans d'autres contextes tels que l'analyse filmique.

Nous faisons référence à l'ouvrage de Bernard Stiegler Etats de choc, Bêtise et savoir au XXIème siècle, Mille et une nuits, jan 2012 
Actually Anthropology, Sociology and all the Humanities and Social Sciences are confronted to the challenge of the digital. A commonly held idea in the United States and named Digital Humanities as generic term is often reduced to the ways Social Sciences and Humanities are using the digital. Our approach in this article offers, referring to the work and notions conceived by B Stiegler, not only a study of but the conception of tools which could give us means and ways to reconsider epistemological foundations of academic disciplines at large. We will take as example an annotative collaborative software of video conceived and used during the ANR project Tic and Tac (2009-2011). Within this software we analyse the creativity process trying to isolate it from the technical capacities of the program. These capacities are questioning: sociological and anthropological methodology of analysis of creativity in a context of innovative technologies. (Project Garage CEA), - creativity process in an annotative technology or during a collaborative editorial development experimented at IRI with the analysis of films.

We refer to the book of Bernard Stiegler Etats de choc, Bêtise et savoir au XXIème siècle, Mille et une nuits, jan 2012

La antropología, la sociología y todas la ciencias humanas y sociales se encuentran hoy en día confrontadas al reto de lo digital. Existe una corriente muy extendida en las Estados-Unidos y definida bajo el término genérico de Digital Humanities que se reduce a menudo a estudiar cómo las SHS utilizan lo digital. Nuestra perspectiva de estudio en este artículo, en cambio, propone, dentro del marco general propuesto por B. Stiegler, no solamente observar sino también concebir herramientas que permitan reconsiderar los fundamentos epistemológicos de las diferentes disciplinas académicas (y no solamente SHS). Nos basaremos en este texto en una plataforma de anotación colaborativa de vídeo utilizada en el marco del proyecto ANR Tic Tac (2009-2011) sobre el análisis de la creatividad, e intentaremos discernir las funciones técnicas de esta herramienta. Estas funciones ponen precisamente en cuestión: la metodología de análisis sociológica o antropológica en términos globales, el proceso de innovación en un contexto de innovación tecnológica (proyecto Garage du CEA), la extensión de la creatividad en la herramienta de anotación así como en las herramientas de editorialización colaborativa experimentadas por el IRI en otros contextos como el del análisis fílmico.

\section{INDEX}

Keywords : indexing, media annotation, contribution, creativity, video, sociologie, collaborative analysis, digital humanities

Palabras claves : indexación, anotación, contribución, creatividad, vídeo, sociología, análisis colaborativo, humanidades digitales

Mots-clés : indexation, annotation, contribution, créativité, vidéo, sociologie, analyse collaborative, humanités numériques

\section{AUTEURS}

\section{VINCENT PUIG}

Institut de recherche et d'innovation (IRI)

vincent.puig@centrepompidou.fr

\section{YVES-MARIE L'HOUR}

Institut de recherche et d'innovation (IRI) - Institut Télécom 